\title{
Aspectos fisiopatológicos de la rehabilitación respiratoria en fibrosis quística
}

\author{
Klgo. Mg. Iván Rodríguez ${ }^{1,3}$, Klgo. Ricardo Arriagada², \\ Dra. Claudia Fuentes ${ }^{1,4}$, Dr. Daniel Zenteno ${ }^{1,4}$ \\ 1 Programa de Rehabilitación Respiratoria Infantil. Servicio de Pediatría, Hospital Guillermo Grant Benavente. Concepción. \\ 2 Unidad de Paciente Crítico. Hospital Higueras. Talcahuano. \\ 3 Escuela de Kinesiología. Facultad de Ciencias de la Salud. Universidad San Sebastián. Concepción. \\ 4 Departamento de Pediatría. Facultad de Medicina. Universidad de Concepción.
}

\section{PATHOPHYSIOLOGY OF RESPIRATORY REHABILITATION IN CYSTIC FIBROSIS}

The Cystic Fibrosis (CF) is an autosomal recessive disease and is triggered by a mutation of the gene coding for cystic fibrosis transmembrane conductance regulator (CFTR) protein; being the respiratory, cardiovascular and musculoskeletal system affected for this mutation, the systemic consequences of CF produce altered physical performance, expressed in less $\mathrm{VO}_{2}$ peak levels which is directly correlated with CF survival rate. In this context, the pulmonary rehabilitation (PR), through aerobic exercise, anaerobic exercise, respiratory muscle training, has shown to be secure and effective in reversing functional decline caused by CFTR mutation. In this paper we review the most important aspects related to exercise pathophysiology and the impact of training on physiological and clinical variables in patients with CF, in order to provide a comprehensive view about of the benefit that the physical exercise could generate in the CF course.

Key words: Cystic fibrosis, pulmonary rehabilitation, pathophysiology, exercise physiology, physical training.

\section{RESUMEN}

La Fibrosis Quística (FQ) es una enfermedad autosómica recesiva y se produce por una mutación del gen que codifica para la proteína reguladora de la conductancia transmembrana (CFTR); siendo el sistema respiratorio, cardiovascular y musculo esquelético afectados por esta mutación. La repercusión multisistémica de la enfermedad, causa alteración en el rendimiento físico, expresado en caídas en el $\mathrm{VO}_{2}$ peak; el que está directamente correlacionado con la sobrevida del paciente portador de FQ. En este contexto la rehabilitación respiratoria (RR), a través del ejercicio aeróbico, anaeróbico, entrenamiento muscular respiratorio y general; ha demostrado ser métodos seguros y efectivos, en la reversión del deterioro funcional causado por la mutación de la CFTR. En la presente trabajo se hace una revisión de la literatura sobre los aspectos más importantes vinculados a la fisiopatología del ejercicio, y el impacto del entrenamiento sobre variables fisiológicas y clínicas en pacientes con FQ, con el fin de proporcionar una mirada integral acerca de los beneficios que potencialmente puede generar el ejercicio en el curso de esta enfermedad.

Palabras clave: Fibrosis quística, rehabilitación respiratoria, fisiopatología, fisiología del ejercicio, entrenamiento físico.

\section{INTRODUCCIÓN}

La Fibrosis Quística (FQ) es la enfermedad hereditaria letal más frecuente en raza blanca, se transmite de manera autosómica recesiva y se produce por una mutación en el gen que codifica la proteína reguladora de la conductancia transmembrana de la FQ (CFTR), ubicado en el brazo largo del cromosoma $7^{(I)}$. De evolución crónica, progresiva y compromiso multisistémico, presenta grandes variaciones fenotípicas y

Correspondencia: Iván Rodríguez Núñez.

Kinesiólogo Coordinador Programa de Rehabilitación Respiratoria infantil,

Servicio de Pediatría, Hospital Guillermo Grant Benavente. Concepción.

Telefono: 56(4I) 2687489.

E-mail: klgo.ivanronu@gmail.com genotipicas según los diversos grupos étnicos estudiados (2). En Chile, de acuerdo a la mezcla racial existente y los estudios de las mutaciones, se estima una incidencia aproximada de I/8.000 a I// 0.000 recién nacidos vivos (RN), lo que significa aproximadamente 30 casos nuevos anuales ${ }^{(1)}$.

La proteína CFTR es un monómero de 1.480 aminoácidos, posee dos grupos de seis dominios trans-membranicos, dos dominios de unión a nucleótido y un dominio regulatorio con sitios específicos de fosforilación y se encuentra ampliamente distribuida en el organismo ${ }^{(3,4)}$. Por lo que desde el punto de vista fisiopatológico, las principales consecuencias sistémicas de la enfermedad son la alteración en el transporte mucociliar, disfunción pancreática exocrina y atrofia muscular intrínseca; lo que desemboca en la disminución del rendimiento físico y deterioro de la calidad de vida(5).

El ejercicio físico ha pasado a formar parte importante en 
el manejo integral de estos pacientes, debido a los beneficios sobre el control de la enfermedad, calidad de vida, pronóstico y mortalidad ${ }^{(6,7)}$. Diversos estudios han mostrado que el rendimiento aeróbico máximo está determinado por la función pulmonar y masa libre de grasa, y además constituye un marcador de sobrevida en pacientes con $\mathrm{FQ}^{(8)}$. Nixon y cols establecieron que pacientes con $\mathrm{VO}_{2}$ peak (máximo consumo de oxígeno alcanzado en un test de esfuerzo) mayor al 83\% del predicho tenían una tasa de sobrevida del $83 \%$ en ocho años, comparado con tasas del $28 \%$ para pacientes con $\mathrm{VO}_{2}$ peak menor al $58 \%$ del predicho( ${ }^{(9)}$. Por otra parte, Moorcroft y cols, concluyeron que si bien es cierto los resultados de los test de ejercicio poseen buena correlación con sobrevida, no son mejores que el VEFI como indicadores pronósticos ${ }^{(10)}$. En adición a eso Pianosi y cols, en un estudio prospectivo, demostró que la tasa de caída del $\mathrm{VO}_{2}$ peak y su valor al final de 8 años de seguimiento, fue significativamente predictor de mortalidad, donde pacientes con valores menores a $32 \mathrm{ml} / \mathrm{kg} /$ min exhibieron un dramático aumento en la mortalidad, en relación a aquellos que superaron los $45 \mathrm{ml} / \mathrm{kg} / \mathrm{min}$ de $\mathrm{VO}_{2}$ peak de los cuales ninguno falleció( (I). Queda claro entonces que el rendimiento físico es muy importante para el pronóstico de del paciente con FQ; sin embargo, la fisiopatología del ejercicio y el impacto de las distintas modalidades de entrenamiento en la tolerancia al ejercicio y curso clínico de la enfermedad no han sido abordadas en conjunto en este grupo de pacientes.

En el presente artículo se hace una revisión de la literatura sobre los aspectos más importantes vinculados a la fisiopatología del ejercicio, y el impacto del entrenamiento sobre variables fisiológicas y clínicas en pacientes con FQ, con el fin de proporcionar una mirada integral acerca de los beneficios que potencialmente puede generar el ejercicio en el curso de esta enfermedad.

\section{FISIOPATOLOGÍA DEL EJERCICIO EN FIBROSIS QUÍSTICA}

\section{Respuesta hemodinámica}

El sustrato fisiopatológico de la respuesta hemodinámica alterada en el paciente con FQ, responde a la alteración histológica de la microvasculatura pulmonar. En este contexto se ha observado la presencia de hipertrofia muscular lisa de la arteria pulmonar, fibrosis y engrosamiento de la pared de venas pulmonares y desaparición de las ramas pequeñas de la arteria pulmonar a medida que la enfermedad progresa. Por otra parte, se ha evidenciado la existencia de correlación significativa del engrosamiento de la pared muscular vascular con el grado de hipertrofia del ventrículo derecho (VD) (12). Esto trae como consecuencia, en mayor o menor medida, la presencia de hipertensión pulmonar (HTP) manifestándose principalmente en estadios severos de la enfermedad y la subsecuente insuficiencia ventricular sistólica y diastólica derecha lo que conlleva al cor pulmonale ${ }^{(13-15)}$. Además la sobrecarga de presión y volumen en el VD produce desplazamiento del septum interventricular hacia la izquierda, y compresión del ventrículo izquierdo $(\mathrm{VI})$ en un fenómeno denominado "interdependencia ventricular", lo que producirá una contracción y dilatación diskinética en el VI, disminuyendo el gasto cardiaco ${ }^{(16)}$.

Hull y cols, recientemente demostraron que pacientes con FQ leve a moderada poseen una respuesta hemodinámica central alterada al ejercicio, evidenciado por un mayor índice de aumento (Alx), parámetro vascular de rigidez arterial y reflexión de onda arterial global, comparado con sujetos control, lo que muestra una carga sistólica central exagerada y un trabajo cardiaco aumentado durante ejercicio a baja intensidad ${ }^{(17)}$. Estas alteraciones hemodinámicas en su conjunto traen como consecuencia una disminución de la capacidad de aumentar el gasto cardiaco para suplir las demandas metabólicas durante el ejercicio, manifestado en la presencia de menor fracción de eyección tanto en VD y $\mathrm{VI}^{(18)}$.

\section{Función muscular esquelética}

Estudios recientes han demostrado que la proteína CFTR está funcionalmente expresada en el músculo esquelético, con una débil expresión en el sarcolema y fuerte expresión en el retículo sarcoplásmico (RS). Donde su desregulación altera el gradiente electroquímico a través de la membrana reticular, y por lo tanto, altera el funcionamiento de algunos canales como el canal/receptor de rianodina (RyR) o la Bomba $\mathrm{Ca}^{+2}$ ATPasa del RS, los cuales son esenciales en el acomplamiento excitación-contracción lo que reduce el movimiento actinamiosina mediado por ATP y activado por $\mathrm{Ca}^{+2}$, contribuyendo a debilidad muscular esquelética e intolerancia al ejercicio observada en estos pacientes. Cabe mencionar que la bomba de $\mathrm{Ca}^{+2}$ del $\mathrm{RS}$ es responsable del secuestro de $\mathrm{Ca}^{+2}$ desde el citosol, una vez que el musculo esta en reposo, por lo que se ha descrito que en miotúbulos CFTR -/- se aprecian aumentados niveles de $\mathrm{Ca}^{+2}$ intracelular después de despolarización inducido por KCL, sugiriendo que la movilización de $\mathrm{Ca}^{+2}$ se encuentra desregulada en células musculares con CFTR deficiente, lo que se manifiesta en un metabolismo muscular alterado durante la relajación post contracción ${ }^{(19)}$.

Wells y cols, establecieron que los pacientes con CF poseen una bioenergética muscular anormal tanto en reposo como en ejercicio, caracterizado por menor ATP intramuscular y una menor relación ATP/Fosfocreatina (PCr) en reposo, además de un menor nivel de acidosis muscular asociado a menores tasas de recuperación de PCr al final del ejercicio; sugiriendo la existencia de un metabolismo aeróbico alterado en pacientes con $\mathrm{FQ}^{(20)}$. En adición a esto, Tu y cols, observaron que el flujo de ATP inducido por acidosis del musculo esquelético, era abolido en presencia de un inhibidor de la proteína CFTR (CFTR -172$)$. El aumento de la concentración de ATP intersticial y su transformación a adenosina durante la contracción muscular, contribuye de manera importante a la hiperemia asociado a ejercicio, fenómeno que se encuentra severamente alterado en el tejido muscular con CFTR anormal(2I) (Figura I).

La ausencia de esta proteína en miotúbulos de diafragma y extremidades se asocia a un fenotipo hiperinflamatorio con sobreexpresión de IL8, NF-kB, MIP-I $\alpha$ y RANTES, en presencia de citoquinas pro-inflamatorias (TNF- $\alpha,|\mathrm{L}-| \alpha \mid \mathrm{IFN}-\gamma$ ) e infección bacteriana con pseudomona. Así como también, sobreexpresión de reguladores claves del sistema ubiquitin- 


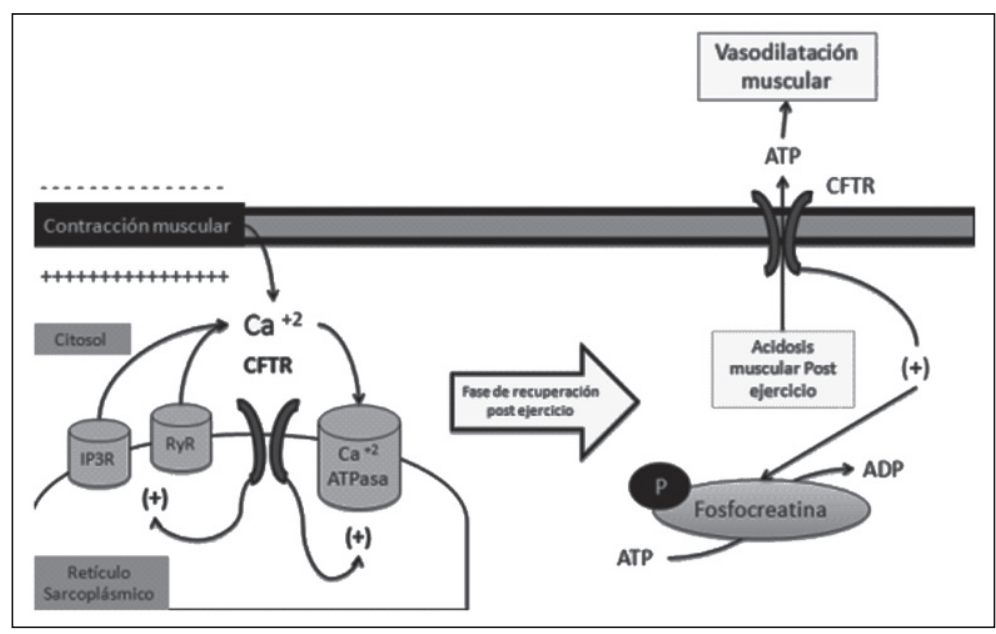

Figura I. Representación esquemática del rol de la CFTR en el musculo esquelético.

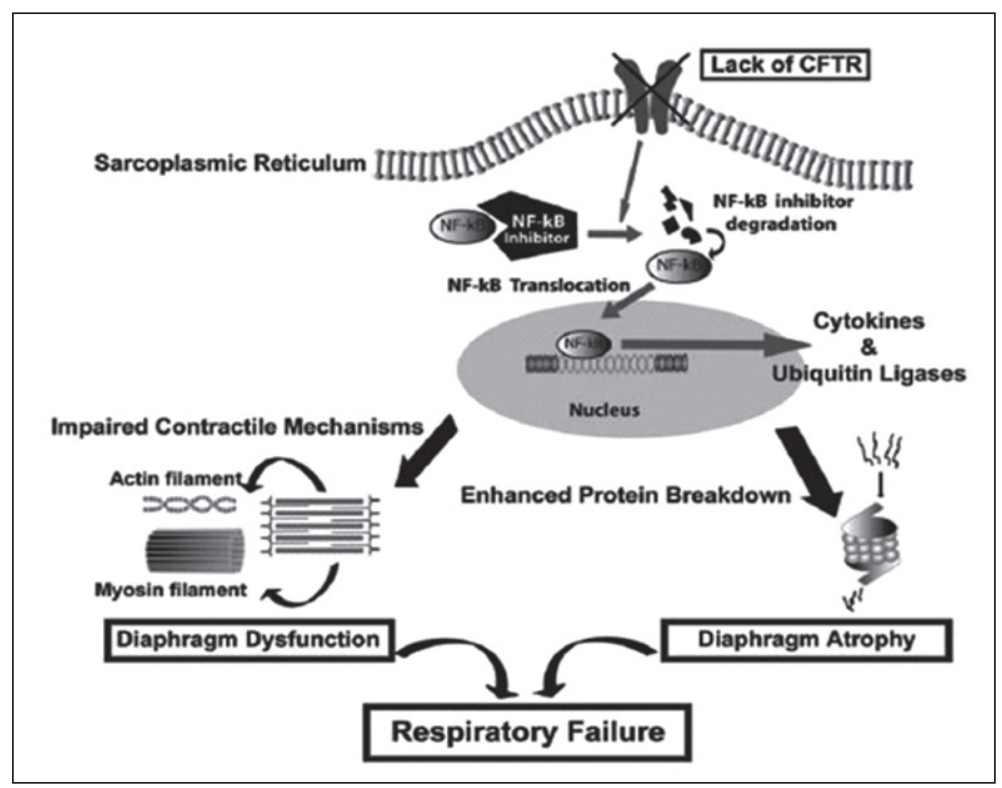

Figura 2. Esquema de la alteración en la modulación inflamatoria causado por la CFTR anormal en el musculo esquelético. Extraído de Divangahi M., Et al., PLoS Genet 2009; 5 (7): el 000586.

proteasoma, vinculado con la pérdida de masa muscular, permitiendo concluir que fibras musculares con CFTR defectuoso exhiben una exagerada sobreregulación de citoquinas y factores inducidores de caquexia durante la infección pulmonar, asociándose a un mayor deterioro de la función diafragmática ${ }^{(22)}$ (Figura 2).

\section{Mecánica respiratoria en ejercicio}

La mecánica respiratoria en ejercicio sufre modificaciones que contribuyen a la eficacia de los músculos respiratorios durante su contracción. Se ha descrito, en sujetos sanos una disminución proporcional del volumen pulmonar al final de la espiración (VPFE) conforme aumenta la intensidad de ejercicio(23,24). Esto permitiría iniciar la contracción diafragmática desde un estado de pre-elongación mayor y así optimizar la relación longitud tensión en beneficio del costo energético de la ventilación durante el ejercicio. Sin embargo, en pacientes con limitación crónica del flujo aéreo, y debido a las mayores constantes de tiempo de su sistema respiratorio, se produce aumento del VPFE lo que condiciona la presencia de hiperinsuflación dinámica constituyendo un factor limitante para la ejecución de ejercicio. En pacientes con FQ, se ha descrito que dicha limitación es dependiente de la severidad de la patología, produciéndose aumentos significativos del VPFE conforme aumenta la intensidad del ejercicio, principalmente en estadios moderados y severos de la enfermedad ${ }^{(25)}$. Sin embargo, estudios recientes han evidenciado que dicha limitación no constituye un factor gatillante de fatiga muscular inspiratoria ni espiratoria post ejercicio(26). No obstante, la hiperinflación estática compromete de manera discreta la capacidad ventilatoria y tolerancia al ejercicio, al ser un predictor de Wpeak y $\mathrm{VO}_{2}$ peak $^{(27)}$.

En conjunto, todos estos resultados demuestran que la limitación ventilatoria del paciente $F Q$ ejerce su influencia sobre la tolerancia al ejercicio más que en la función muscular respiratoria, debido a que la presencia de hiperinflación dinámica compromete la performance en ejercicio sin alterar la fuerza contráctil del grupo muscular inspiratorio y/o espiratorio durante la ejecución del ejercicio.

\section{Impacto de la FQ en el rendimiento físico}

Las principales repercusiones de estas anomalías bioquímicas y mecánicas son la debilidad muscular y la intolerancia al ejercicio. Se ha demostrado que la capacidad máxima de trabajo en relación a la masa magra $\mathrm{VO}_{2}$ máx/FFM y Wmáx/FFM) y fuerza muscular se encuentra significativamente reducida en pacientes con FQ comparado con sujetos sanos, además estos pacientes presentan un mayor costo de oxigeno durante el esfuerzo al presentar una mayor ventilación minuto durante ejercicio submáximo y una alta correlación entre $\mathrm{VO}_{2}$ y carga de trabajo ${ }^{(28)}$. En este contexto, Troosters y cols, demostraron, en sujetos adultos con $\mathrm{FQ}$, que la inactividad física es un factor que contribuye de manera significativa a la tolerancia al ejercicio y debilidad muscular, no obstante, la magnitud de este deterioro es mayor al esperado por la sóla inactividad física ${ }^{29)}$. En relación a la función muscular respiratoria, se ha observado que pacientes con FQ poseen valores de presión inspiratoria (PiMax) y espiratoria (PeMax) bajo lo esperado para la edad, teniendo como principal consecuencia el aumento de la disnea y el $\mathrm{CO}_{2}$ antes, durante y después del ejercicio ${ }^{(30,31)}$ (Figura 3). 


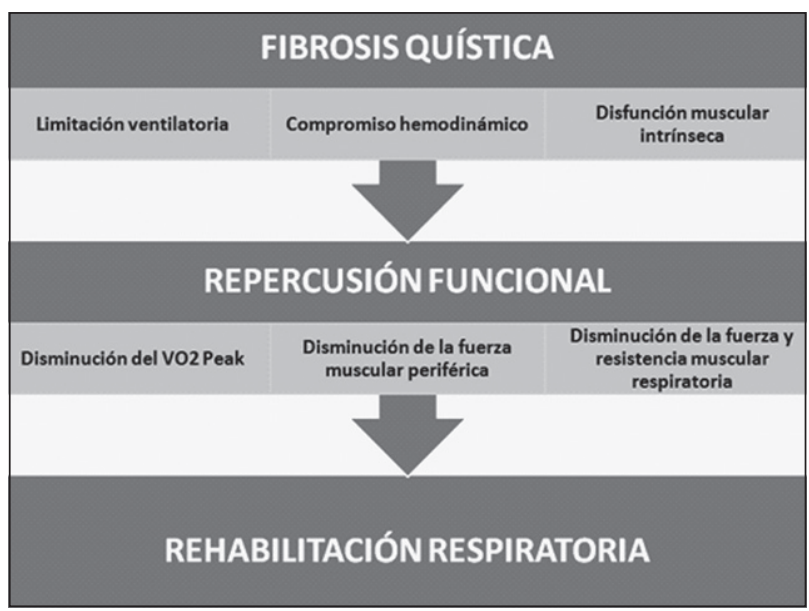

Figura 3. Esquema del compromiso funcional causado por la fibrosis quística.

\section{EFECTO DEL ENTRENAMIENTO EN LA REVERSIÓN DEL DETERIORO FUNCIONAL}

\section{Efecto del entrenamiento aeróbico}

Shoemaker y cols ${ }^{(32)}$, en una revisión sistemática, muestra que el ejercicio aeróbico mejora la capacidad física a través de aumentos significativos del $\mathrm{VO}_{2}$ peak y fuerza, así como también menores niveles de lactato y frecuencia cardiaca de trabajo. Respecto a la función pulmonar, si bien es cierto no observaron mejoras significativas, no obstante, se aprecia una tendencia a mitigar el deterioro con el curso de la enfermedad ${ }^{(32)}$. Similares resultados han sido observados durante la fase de reagudización, donde se ha evidenciado que el entrenamiento aeróbico logra mejorar calidad de vida y función física ${ }^{(33)}$. Estudios recientes han reportado que la adición de técnicas de clearence bronquial al entrenamiento aeróbico también contribuye a mejorar cualidades físicas de fuerza, resistencia y velocidad ${ }^{(34)}$. Lo que demuestra que este método de entrenamiento es una estrategia segura y efectiva para mejorar el performance físico del paciente con FQ.

Desde el punto de vista fisiopatológico se ha determinado el rol inmunomodulador del ejercicio aeróbico ${ }^{(35)}$. En este ámbito, la proteína C-reactiva (PCR) ha mostrado tener correlación significativa con la función pulmonar y el rendimiento físico, así como también con adaptación fisiológica al ejercicio, observándose disminuciones de la PCR en respuesta al entrenamiento aeróbico en pacientes con $\mathrm{FQ}^{(36,37)}$. Ploeger y cols en una revisión sistemática donde integró el efecto del ejercicio agudo y crónico sobre marcadores inflamatorios en diversas patologías, establece que en pacientes con enfermedad inflamatoria crónica, el ejercicio podría gatillar diferentes respuestas inflamatorias según tipo de ejercicio siendo exagerada en ejercicio agudo y atenuada posterior a un programa de entrenamiento. Específicamente en los pacientes con $\mathrm{FQ}$, se han descrito aumentos del TNF- $\alpha$ e IL-6 posterior a 10 intervalos de 2 min de ejercicio al 50\% de $\mathrm{VO}_{2}$ Max, así como, aumento del número total de leucocitos y granulocitos una hora posterior al ejercicio físico ${ }^{(35)}$.

En adición a esto, se ha descrito que el ejercicio aeró- bico modifica las propiedades del esputo y la capacidad de expectoración del moco bronquial, donde se ha registrado que protocolos de entrenamiento aeróbico (en treadmil y cicloergómetro) aumentan significativamente los flujos y volúmenes respiratorios, permitiendo optimizar el proceso de eliminación de secreciones bronquiales ${ }^{(38,39)}$. Incluso protocolos realizados en treadmil disminuyen la impedancia mecánica del esputo debido a la oscilación del tronco asociado a la marcha durante el entrenamiento(40). En la tabla I, se muestra los efectos del ejercicio sobre las variables de rendimiento físico en pacientes con $\mathrm{FQ}$.

\section{Efecto del entrenamiento anaeróbico}

Considerando los efectos benéficos que tiene el entrenamiento anaeróbico tanto en sujetos sanos como en pacientes con diversas patologías, es escasa la evidencia empírica que estudia la actividad anaeróbica en los sujetos portadores de

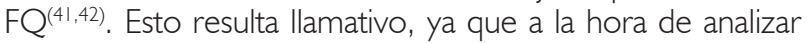
el tipo e intensidad de las actividades que predominan en las actividades diarias de un niño, resultan ser, los esfuerzos de corta duración y alta intensidad, las que se ejecutan con mayor frecuencia ${ }^{(43)}$. Sin embargo, el paciente con FQ se caracteriza por tener un rendimiento limitado en intensidades altas de ejercicio, principalmente debido a las repercusiones nutricionales y respiratorias de la enfermedad ${ }^{(44-46)}$.

Se ha demostrado que 12 semanas de entrenamiento anaeróbico permiten mejoras significativas en capacidad anaeróbica, capacidad aeróbica y calidad de vida ${ }^{(47)}$. Sin embargo, aún queda por dilucidar el impacto de esta modalidad de ejercicio sobre variables fisiológicas y su rol en la reversión del deterioro funcional en estos pacientes.

\section{Efecto del entrenamiento muscular periférico}

El entrenamiento de fuerza (EF) en población pediátrica ha sido materia de debate en las últimas décadas, debido a la importancia de la biodisponibilidad de hormonas sexuales circulantes para el logro de los efectos fisiológicos en respuesta a este método de entrenamiento. Sin embargo, gran parte de la evidencia reciente muestra que el EF es seguro en todas las fases del crecimiento si existe una adecuada supervisión, programa adecuado para la edad y progresiones graduales estandarizadas ${ }^{(48)}$. Behringer y cols en un meta-análisis donde

Tabla I. Efecto del ejercicio sobre variables de rendimiento físico en pacientes con fibrosis quística

\begin{tabular}{|ll|}
\hline Método de entrenamiento & Mejora \\
\hline Aeróbico & $\begin{array}{l}\mathrm{VO}_{2} \mathrm{Máx}, \mathrm{VO}_{2} \text { Peak, Clearence } \\
\text { bronquial. Fuerza de EEll, calidad } \\
\text { de vida }\end{array}$ \\
\hline $\begin{array}{l}\mathrm{VO}_{2} \text { Máx, } \mathrm{VO}_{2} \text { Peak, Fuerza de } \\
\text { EEll, calidad de vida }\end{array}$ \\
$\begin{array}{ll}\text { Entrenamiento muscular } \\
\text { general }\end{array}$ & Fuerza, Resistencia \\
$\begin{array}{l}\text { Entrenamiento muscular } \\
\text { respiratorio }\end{array}$ & $\begin{array}{l}\text { Fuerza y resistencia muscular res- } \\
\text { piratoria, capacidad vital, calidad } \\
\text { de vida, ventilación en ejercicio? }\end{array}$ \\
\hline
\end{tabular}


revisa los efectos del entrenamiento de fuerza en niños y adolescentes, muestra que el EF es efectivo en niños, y que la entrenabilidad de la fuerza muscular aumenta levemente con la edad lo que hace que la madurez sea un importante predictor de resultados de entrenamiento. Además encontró que las ganancias de la fuerza muscular son dependientes del volumen de entrenamiento en términos del número de sesiones por semana y duración del programa de intervención, lo que demuestra que en niños este método de entrenamiento es una vía viable para mejorar la función muscular(49).

Es escasa la evidencia que evalúa los efectos aislados del EF en pacientes con FQ, debido a que habitualmente se encuentra incorporado dentro de los protocolos de RR integrales en el manejo de estos pacientes. Orenstein y cols, diseñaron un estudio para evaluar los efectos diferenciales entre entrenamiento de fuerza y aeróbico donde demostraron que ambos tipos de entrenamiento fueron asociados con aumentos de fuerza y capacidad de trabajo físico, sin embargo, sólo el EF aumentó la fuerza máxima del bíceps. Más tarde, Sahlberg y cols, no observó mejoras significativas en la fuerza muscular argumentando que en este grupo de pacientes existe una alteración muscular fisiológica que se correlaciona con el deterioro de la función pulmonar. Es necesario futuros estudios para dilucidar el real impacto sobre variables clínicas de este tipo de entrenamiento. Por ahora, queda claro que la RR en FQ debe estar enmarcado bajo el principio de la integralidad, donde se deben combinar una variada gama de estrategias de intervención, donde el EF asume un rol fundamental para mitigar el deterioro causado por el desbalance bioquímico asociado a la disfunción de la CFTR en el musculo esquelético.

\section{Efectos del entrenamiento muscular respiratorio}

La limitación al flujo aéreo, alteración progresiva del intercambio gaseoso, parénquima pulmonar con presencia de tejido cicatricial y pérdida del potencial elástico, que conlleva al fenómeno de hiperinsuflación pulmonar forma parte del sustrato fisiopatológico que sustenta el deterioro respiratorio del paciente con $\mathrm{FQ}^{(50)}$. Todo lo anterior genera una sobrecarga hacia el grupo muscular respiratorio, quién debe lograr generar cambios de presión, a pesar de las deficiencias de su eficacia mecánica y un inadecuado estado nutricional lo que propicia la atrofia muscular, aspectos que han sido evidenciados de forma significativa en la musculatura periférica y respiratoria de éstos pacientes ${ }^{(51)}$.

Se han descrito diversos métodos de EMR, según tipo, frecuencia, intensidad y duración ${ }^{(52)}$. En este contexto, Jong y cols evidenció que 6 semanas de entrenamiento muscular respiratorio al $40 \%$ de la presión inspiratoria máxima (Pimax) permite aumentos significativos en la resistencia muscular inspiratoria, sin variaciones en las variables de función pulmonar ${ }^{(53)}$. Más tarde, Enright y cols evaluó el impacto de la intensidad de entrenamiento demostrando que el EMR desarrollado a alta intensidad (80\% Pimax), permite mejoras significativas en la capacidad vital, en la resistencia y fuerza muscular inspiratoria; así como también, en la ansiedad y depresión, evaluados por la escala "Hospital Anxiety and Depression"(54).

Hasta ahora, la evidencia respalda el empleo de altas intensidades de entrenamiento debido a su impacto sobre la fuerza y resistencia muscular respiratoria, así como en los volúmenes pulmonares del paciente portador de $\mathrm{FQ}^{(55)}$. Sin embargo, dado que el EMR forma parte de los planes de RR integral del paciente con FQ y la escasa evidencia del entrenamiento especifico de músculos respiratorio en este grupo de pacientes, es imposible establecer beneficios funcionales, más allá del solo aumento de la función muscular respiratoria. Sin embargo, es claro que adecuados niveles de fuerza y resistencia de los músculos respiratorios son necesarios para una adecuada respuesta ventilatoria al ejercicio lo que puede

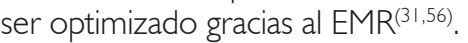

\section{CONCLUSIÓN}

La rehabilitación respiratoria ha adquirido un rol fundamental en el manejo de la patología respiratoria crónica pediátrica. En este contexto, y dada la compleja fisiopatología de las repercusiones extrapulmonares de la FQ y el impacto de los efectos fisiológicos del ejercicio sobre el curso clínico de la enfermedad, existe sustrato fisiopatológico para abalar las distintas modalidades de entrenamiento físico como coadyuvantes a la terapia farmacológica. En el presente trabajo, se revisaron los aspectos más relevantes vinculados con el deterioro funcional generado a consecuencia de la mutación de la proteína CFTR, y el rol que las distintas modalidades de ejercicio tiene sobre estas alteraciones. Futuros estudios que evalúan el impacto del ejercicio físico sobre el control de la enfermedad y sobrevida son necesarios, para apoyar la evidencia fisiopatológica que respalda el beneficio del ejercicio en revertir las alteraciones funcionales en los pacientes portadores de FQ.

\section{REFERENCIAS}

I. MINSAL. Programa Nacional de Fibrosis Quística. Orientaciones Técnicas Programáticas Para Diagnóstico y Tratamiento. Santiago : s.n., 2012. (Extraído de http://fibrosisquisticachile.cl/ el día 23 de octubre de 2012).

2. Vega Briceño L, Sánchez I. Fibrosis quística: Actualización en sus aspectos básicos. Rev Chil Pediatr 2005; 76: 464-70.

3. Morales M, Capella M, Lopes A. Structure and function of the cystic fibrosis transmembrane conductance regulator. Braz | Med Biol Res 1999; 32: 1021-8.

4. Schwiebert E, Benos D, Egan M, Stutts M, Guggino W. CFTR Is a conductance regulator as well as chloride channel. Physiol Rev 1999; 79 (Suppl I): SI 46-SI62.

5. Boucher R. New concepts of the pathogenesis of cystic fibrosis lung disease. Eur Respir J 2004; 23: 146-58.

6. Rand S, Prasad A. Exercise as part of a cystic fibrosis therapeutic routine. Expert Rev Respir Med 20 12; 6: 341-52.

7. Hebestreit $H$, Kieser $S$, Junge $S$, et al. Long-term effects of a partially supervised conditioning programme in cystic fibrosis. Eur Respir J 20 10; 35: 578-83.

8. Klijin P, van der Net J, Kimpen J, van der Ent C. Longitudinal determinants of peak aerobic performance in children with cystic fibrosis. Chest 2003; 124: 2215-9.

9. Nixon P, Orenstein D, Kelsey S. The prognostic value of exercise 
testing in patients with cystic fibrosis. N Engl J Med 1992; 327: 1785-8.

10. Moorcroft A J, Dodd M E, Webb A K. Exercise testing and prognosis in adult in cystic fibrosis. Thorax 1997; 52: 291-3.

1 I. Pianosi P, LeBlanc J, Almudevar A. Peak oxygen uptake and mortality in children with cystic fibrosis. Thorax 2005; 60: 50-4.

12. Ryland D, Reid L. The pulmonary curculation in cystic fibrosis. Thorax 1975; 30: 285-92.

13. Florea VG, Florea ND, Sharma R. Right ventricular dysfunction in adult severe cystic fibrosis. Chest 2000; I I 8: I063-8.

14. Baño-Rodrigo A, Salcedo-Posadas A, Villa-Asensi J, et al. Right ventricular dysfunction in adolescents with mild cystic fibrosis. J Cyst Fibrosis 2012; 11: 274-80.

15. Ozcelik N, Shell R, Holtzlander M, Cua C. Decreased Right Ventricular Function in Healthy Pediatric Cystic Fibrosis Patients Versus Non-Cystic Fibrosis Patients. Pediatr Cardiol 2012.

16. Jacobstein M, Hirschfeld S, Winnie G, et al. Ventricular interdependence in severe cystic fibrosis. A two-dimensional echocardiographic study. Chest 1981; 80: 399-404.

17. Hull J, Ansley L, Bolton C, et al. The effect of exercise on large artery haemodynamics in cystic fibrosis. Cyst Fibros 2011; 10: | $21-7$.

18. Bright-Thomas R, Webb K. The heart in cystic fibrosis. J R Soc Med 2002; 95 (Suppl 4I): 2- 10.

19. Lamhonwah A, Bear C, Huan L, et al. Cystic fibrosis transmembrane conductance regulator in human muscle dysfunction causes abnormal metabolic recovery in exercise. Ann Neurol 20 10; 67: 802-8.

20. Wells G, Wilkes D, Scheiderman J, et al. Skeletal muscle metabolism in cystic fibrosis and primary ciliary dyskinesia. Pediatr Res 2011; 69: 40-5.

21. Tu J, Le G, Ballard H. Involvement of the cystic fibrosis transmembrane conductance regulator in the acidosis-induced efflux of ATP from rat skeletal muscle. J Physiol 20 I0; 588: 4563-78.

22. Divangahi M, Balghi H, Danialou G, et al. Lack of CFTR in Skeletal Muscle Predisposes to Muscle Wasting and Diaphragm Muscle Pump Failure in Cystic Fibrosis Mice. PLoS Genet 2009; 5: el000586.

23. Henke K, Sharratt M, Peqelow D, Dempsey J. Regulation of endexpiratory lung volume during exercise. J Appl Physiol 1988; 64: 135-46.

24. Guenette J, Witt J, Mckenzie D, Road J, Sheel A. Respiratory mechanic during exercise in endurance-trained men and women. J Physiol 2007; 582: 1309-22.

25. Alison J, Regins J, Donnelly P, Adams R, et al. End-expiratory lung volume during arm and leg exercise in normal subjects and patients with cystic fibrosis. Am J Respir Crit Care Med 1998; 158 (5 Pt I): 1450-8.

26. Reilly $\mathrm{C}$, Ward $\mathrm{K}$, Jolley $\mathrm{C}$, et al. Effect of endurance exercise on respiratory muscle function in patients with cystic fibrosis. Respir Physiol Neurobiol 20 I 2; 180: 316-22.

27. Werkman $M$, Hulzebos $H$, Arets $H$, et al. Is static hyperinflation a limiting factor during exercise in adolescents with cystic fibrosis. Pediatr Pulmonol 201 I; 46: I19-24.

28. de Meer K, Gulmans V, van der Laag J. Peripheal muscle weakness and exercise capacity in children with cystic fibrosis. Am J Respir Crit Care Med 1999; 159: 748-54.

29. Troosters T, Langer D, Vrijsen B. Skeletal muscle weakness, exercise tolerance and physical activity in adults with cystic fibrosis.
Eur Respir J 2009; 33: 99- 106

30. Rodríguez I, Zenteno D, Fuentes C, et al. Rehabilitación respiratoria en un hospital publico de Chile. Neumol Pediatr 20 I I; 6: 170.

31. Leroy S, Pérez T, Neviere R, Aguilaniu B, Wallaert B. Determinants of dyspnea and alveolar hypoventilation during exercise in cystic fibrosis: Impact of inspiratory muscle endurance. J Cyst Fibros 2011; 10: 159-65.

32. Shoemaker M, Hurt H, Arndt L. The Evidence Regarding Exercise Training in the Management of Cystic Fibrosis: A Systematic Review. Cardiopulm Phys Ther J 2008; 19: 75-83.

33. Selvadurai $H$, Blimkie C, Meyers N, et al. Randomized controlled study of in-hospital exercise training programs in children with cystic fibrosis. Pediatr Pulmonol 2002; 33: 194-200.

34. Elbasan B, Tunali N, Duzqun I, Ozcelik U. Effects of chest physiotherapy and aerobic exercise training on physical fitness in young children with cystic fibrosis. Ital J Pediatr 2012; 38: 2- I0.

35. Ploeger $H$, Takken T, de Greef $M$, Timmons B. The effects of acute and chronic exercise on inflammatory markers in children and adults with a chronic inflammatory disease: a systematic review. Exerc Immunol Rev 2009; I 5: 6-4I.

36. Levy H, Kalish L, Huntington I, et al. Inflammatory markers of lung disease in adult patients with cystic fibrosis. Pediatr Pulmonol 2007; 42: 256-62.

37. de Oliveira A, de Oliveira JC, Mesquita-Ferrari R. Inflammatory process modulation in children with cystic fibrosis submitted to aerobic training. Arch Med Sci 2009; 5: 422-6.

38. Salh W, Bilton D, Dodd $M$, Webb A. Effect of exercise and physiotherapy in aiding sputum expectoration in adults with cystic fibrosis. Thorax 1989; 44: 1006-8.

39. Badwin D, Hill A, Peckham D, Konx A. Effect of addition of exercise to chest physiotherapy on sputum expectoration and lung function in adults with cystic fibrosis. Respir Med 1994; 88: 49-53.

40. Dwyer TJ, Alison JA, McKeough ZJ, et al. Effects of exercise on respiratory flow and sputum properties in patients with cystic fibrosis. Chest 2011; 139: 870-7.

41. Corte de Araujo AC, Roschel H, Picanço AR. Similar health benefits of endurance and high-intensity interval training in obese children. PLoS One 2012; 7: e427-47.

42. Dunham, C y Harms, CA. Effects of high-intensity interval training on pulmonary function. Eur J Appl Physiol 20 I2; I 12: 306 I-8.

43. Bailey RC, Olson J, Pepper SL. The level and tempo of children's physical activities: An observational study. Med Sci Sports Exerc 1995; 27: 1033-41.

44. Boas, SR, Danduran, MJ, McColley, SA. Energy metabolism during anaerobic exercise in children with cystic fibrosis and asthma. Med Sci Sports Exerc 1999; 31: 1242-9.

45. Boas SR, Joswiak ML, Nixon PA, Fulton JA, Orenstein DM. Factors limiting anaerobic performance in adolescent males with cystic fibrosis. Med Sci Sports Exerc 1996; 28: 291-8.

46. Klijn PH, Terheggen-Lagro SW, Van Der Ent CK. Anaerobic exercise in pediatric cystic fibrosis. Pediatr Pulmonol 2003; 36: 223-9.

47. Klijn P, Oudshoorn A, van der Ent C.Effects of Anaerobic Training in Children With Cystic Fibrosis. A randomized controlled study. Chest 2004; 125: 1299-305.

48. Matos, N, Winsley R. Trainability of young athletes and overtraining. J Sport Sci Med 2007; 6: 353-67.

49. Behringer M, Vom Heede A, Yue Z, Mester J. Effects of resistance 
training in children and adolescents: A Meta-analysis. Pediatrics 2010; 126: el199-el210.

50. Konstan MW, Berger M. Current understanding of the inflammatory process in cystic fibrosis: onset and etiology. Pediatr Pulmonol 1997; 24: 137-42.

5।. Pinet C, Cassart M, Scillia P. Function and bulk of respiratory and limb muscles in patients with cystic fibrosis. Am J Respir Crit Care Med 2003; 168: 989-94.

52. Reid WD, Geddes EL, Brooks D, O’ Brien K, Crowe J. Inspiratory muscle training in COPD. Special series on Skeletal Muscle Training. Physiother Can 2004; 56: I28-42.
53. Jong W, Van Aalderen W, Kraan J, et al. Inspiratory muscle Training in Patients With Cystic Fibrosis. Respir Med 200 I; 95: 31 -6.

54. Enright S, Chatham K, Ionescu AA, Unnithan VB, Shale DJ. Inspiratory muscle training improves lung function and exercise capacity in adults with cystic fibrosis. Chest 2004; 126: 405-I I.

55. Reid WD, Lynne G, O'Brien K, Brook D, Crowe J. Effects of inspiratory muscle training in cystic fibrosis: A systematic review. Clin Rehabilitation 2008; 22: 1003-13.

56. Dunnink MA, Doeleman WR, Trappenburg JC, de Vries WR. Respiratory muscle strength in stable adolescent and adult patients with cystic fibrosis. J Cyst Fibros 2009; 8: 31-6. 